\title{
History and Practice of Thyroid Fine-Needle Aspiration in China, Based on Retrospective Study of the Practice in Shandong University Qilu Hospital
}

\author{
Zhiyan Liu ${ }^{1,2} \cdot$ Dongge Liu ${ }^{3,4}$ \\ Bowen $\mathrm{Ma}^{5} \cdot$ Xiaofang Zhang ${ }^{1,2}$ \\ Peng Su ${ }^{1} \cdot$ Li Chen $^{6} \cdot$ Qingdong Zeng \\ ${ }^{1}$ Department of Pathology, School of Basic \\ Medical Science, Shandong University, Jinan; \\ 2Department of Pathology, Shandong University \\ Qilu Hospital, Jinan; ${ }^{3}$ Department of Pathology, \\ Beijing Hospital, Beijing; ${ }^{4}$ Chinese Cytology \\ Association, Beijing: ${ }^{5}$ Department of \\ Cytopathology, Cancer Hospital of Xinjiang \\ Medical University, Wulumuqi: ${ }^{6}$ Department of \\ Endocrinology, Shandong University Qilu \\ Hospital, Jinan; ${ }^{7}$ Department of General Surgery, \\ Shandong University Qilu Hospital, Jinan, China \\ Received: July 26, 2017 \\ Revised: September 8, 2017 \\ Accepted: September 12, 2017

\section{Corresponding Author} \\ Zhiyan Liu, MD, PhD \\ Department of Pathology, School of Basic Medical \\ Science, Shandong University, Jinan, Shandong, \\ China \\ Tel: +86-18560081167 \\ Fax: +86-53182679225 \\ E-mail: zhiyanliu@sdu.edu.cn
}

Cytology in China developed from nothing and underwent a long journey from gynecologic cytology to that of all organs, laying a solid foundation for new developments in the 21st century. Thyroid fine-needle aspiration (FNA) was primarily developed in an endocrinology department and then in the clinical laboratory department or pathology department in the 1970-80s. Wrights staining is popular in endocrine and clinical laboratory departments, while hematoxylin and eosin staining is common in pathology. Liquid based cytology is not common in thyroid FNA cytology, while $B R A F^{\mathrm{V} 600 \mathrm{E}}$ mutation analysis has been the most popular molecular test. The history and practice of thyroid FNA practice in China were reviewed based on retrospective study of the practice in Qilu Hospital of Shandong University.

Key Words: Thyroid fine needle aspiration; Practice; China; Qilu Hospital

\section{THE BRIEF HISTORY OF CHINESE CYTOPATHOLOGY}

In the 1950s, Dr. Dawang Yang returned to China to start cervical cytology after completing her academic studies in the United States. ${ }^{1,2}$ She began the practice of Papanicolaou cervical smear classification and a cervical cancer screening program in Peking Union Hospital. ${ }^{3}$ Vaginal Cytopathology was published by Dr. Dawang Yang in 1952, which was the first Chinese cytology book and marked the start of modern cytopathology in China.

Esophageal balloon cytology was developed for screening of esophageal cancer, and a series of English publications from China made it well known around the world since the 1960s. ${ }^{4-6}$
Fine-needle aspiration (FNA) was applied first on the body surface and then in deep organs in the 1970-80s. Bone tumor cytodiagnosis was developed by hematologist and cytologist Dr. Xiaojing Peng, ${ }^{7}$ who published the first Chinese FNA book, Atlas of Clinical Cytology, in 1972. The Chinese Academy of Cytology was founded and the first National Clinical Cytology Conference was held in 1985, which was a milestone of cytology in China. ${ }^{8}$

New ancillary techniques such as immunocytochemistry, flow cytometry and DNA alteration analysis were applied in addition to cytopathology starting at the end of 1980s. ${ }^{1,9}$ Liquidbased cytology was initially applied to the cervical smear in the 1990s and greatly improved slide quality and accuracy of diagnosis. The Bethesda System (TBS) for reporting cervical cytology replaced 
Papanicolaou classification, and computer-aided analysis started to play a role.

Cytology has started to play a more prominent role in diagnosis, and quality control of cytology has further improved during the recent 10 years. The Cytology Operational Manual and Quality Control Standards were proposed by the Cytology Section of the Chinese Pathology Association in 2007. A Cytology Quality Control Expert Team was formed in 2010 to supervise clinical diagnosis and academic training, and international exchanges became more popular.

\section{THYROID FINE-NEEDLE ASPIRATION IN CHINA}

Thyroid FNA was primarily developed in endocrinology departments and became popular around China in the 1970-80s. Wright's stain was originally the most popular staining method and was founded by hematologist and cytologist Dr. Xiaojing Peng. In 1987, a national conference posited that FNA cytology should be a branch of pathology, and the cytologist should have a background of surgical pathology. Thyroid FNA began to increase in popularity in some pathology departments, and hematoxylin and eosin staining was applied because it increased the ease of comparing histological samples. Ultrasound-guided thyroid FNA (UG-FNA) became popular gradually, and molecular examination was applied as an additional diagnostic method for papillary thyroid carcinoma (PTC).

\section{MOLECULAR TESTING OF THYROID FINE-NEEDLE ASPIRATION IN CHINA}

Next-generation sequencing was proposed recently to improve the diagnosis of thyroid FNA specimens with indeterminate cytology; however, it is expensive and not well accepted in clinics in China. ${ }^{10-12} B R A F^{\mathrm{V} 600 \mathrm{E}}$ mutation analysis was recommended by American Thyroid Association (ATA) guidelines as an auxiliary diagnostic method for thyroid FNA cytology. ${ }^{13,14}$ The amplification refractory mutation system is efficient and inexpensive and is the most popular method for detection of $B R A F^{\mathrm{V} 600 \mathrm{E}} \mathrm{mu}-$ tation in China. Zhang et al. ${ }^{14}$ recently studied the Thyroid Imaging Reporting and Data System (TIRADS), Bethesda System for Reporting Thyroid Cytopathology (BSRTC), and the BRAF $F^{\mathrm{V} 600 \mathrm{~B}}$ mutation analysis as molecular tools in diagnosing thyroid carcinoma. The TIRADS was applied for selecting patients for FNA biopsy and $B R A F^{\mathrm{V} 600 \mathrm{E}}$ mutation analysis. They found that $B R A F^{\mathrm{V} 600 \mathrm{E}}$ mutation detection had the best sensitivity, specificity, and accuracy among the three methods. Both TIRADS and
$B R A F^{\mathrm{V} 600 \mathrm{E}}$ detection showed increased sensitivity and accuracy when combined with BSRTC. Of all methods, a combination of BSRTC and $B R A F^{\mathrm{V} 600 \mathrm{E}}$ mutation detection demonstrated the best diagnostic efficiency. ${ }^{14}$

\section{PRACTICE IN QILU HOSPITAL, SHANDONG PROVINCE}

Thyroid FNA is not yet well accepted in China, and most general hospitals use frozen sectioning as a diagnostic method instead of thyroid FNA. Some hospitals began to use UG-FNA around the 1990s, along with diagnostic frozen sectioning. UG-FNA is more popular in local hospital than in general hospital. Qilu Hospital is one of the epitomes of thyroid FNA in China, and began performing non-ultrasound guided thyroid FNA cytology in the endocrinology department (including both FNA and cytopathology) in 1991. UG-FNA for thyroid nodules began in 2014, the pathologist began to sign the thyroid FNA report instead of the endocrinologist.

Another point to note is that most Chinese patients choose to undergo diagnostic surgery in the presence of unfavorable clinical and ultrasonographic features, no matter what the size is. There were 2,612 thyroid surgeries, all with a rapid intraoperative pathological diagnosis using diagnostic frozen section, from 2015 to 2016 in our department. ${ }^{15}$ However, only 791 thyroid FNA patients (30.3\%) underwent thyroid surgery. This indicates that there is still ample opportunity for advancement of thyroid FNA in China. Thyroid FNA should be performed to avoid unnecessary surgery for benign thyroid lesions.

\section{Materials and methods}

A retrospective study was conducted of all patients with UGFNA between January 2014 and April 2017 in Qilu Hospital, Shandong University. All patients had available thyroid ultrasound records. Approval was obtained from Qilu Hospital ethics committee, and the patients provided written informed consent. Criteria for FNA were those of Dr. Zhu et al. ${ }^{16}$ Hematoxylin and eosin-stained slides of all tumors were reviewed by three pathologists (Z.L., X.Z., and P.S.). Diagnosis was made according to BSRTC, as shown in Table 1; the only exception was that the cystic-only group was classified into the benign group rather than as nondiagnostic. ${ }^{17}$

\section{Criteria for surgical treatment}

Thyroid surgery was recommended to all patients with suspicion of malignancy or malignancy reports. Diagnostic surgery and 
frozen sectioning were suggested for patients with high-risk clinical or ultrasonographic features. Patients with at least one atypia of undetermined significance or follicular lesion of undetermined significance (AUS/FLUS) report were recommended for repeat FNA or diagnostic partial thyroid lobectomy. Once malignant histological evidence was demonstrated by frozen section, lobectomy was performed for papillary thyroid microcarcinoma (PTMC), and total thyroidectomy was usually performed for tumors larger than $1 \mathrm{~cm}$ with lymph node metastasis. Patients refusing surgical treatment or with high surgical risk were recommended for clinical and ultrasonographic follow-up. Patients with benign FNA cytology diagnosis underwent surgery only when there were clinical symptoms. Patients with nondiagnostic reports were recommended for repeat FNA or clinical and ultrasonographic follow-up.

\section{Results and discussion}

As shown in Table 1, FNA performed on 2838 thyroid nodules showed $3.6 \%$ nondiagnostic specimens, $44.7 \%$ benign nodules, $7.1 \%$ indeterminate $(6.9 \%$ AUS/FLUS and $0.2 \%$ follicular neoplasm or suspicious for a follicular neoplasm [FN/SFN]), $14.1 \%$ suspicious for malignancy, and $30.6 \%$ positive for malignancy. The correlation between FNA cytology and histological diagnosis is shown in Table 2.

Table 1. Results of FNA cytology according to the Bethesda System for reporting thyroid FNA

\begin{tabular}{lc}
\hline Bethesda category & Nodule \\
\hline Nondiagnostic & $101(3.6)$ \\
Benign & $1,268^{\mathrm{a}}(44.7)$ \\
AUS/FLUS & $195(6.9)$ \\
FN/SFN & $5(0.2)$ \\
Suspicious for malignancy & $401(14.1)$ \\
Malignancy & $868(30.6)$ \\
Total & $2,838(100)$ \\
\hline
\end{tabular}

Values are presented as number (\%).

FNA, fine-needle aspiration; AUS/FLUS, atypia of undetermined significance or follicular lesion of undetermined significance; FN/SFN, follicular neoplasm or suspicious for a follicular neoplasm.

a151 cyst fluid only.
The most common FNA diagnosis rendered in our practice was benign, which was nearly the same as the Bethesda expected incidence. Ninety cases of benign nodules were followed with clinical management because of high-risk ultrasound results, 31 cases were proved to be PTMC less than $5 \mathrm{~mm}$ in diameter, and one case was mucosa-associated B-cell lymphoma. This positive rate of FNA for PTMC less than $5 \mathrm{~mm}$ is worse than other practices in China, probably due to the unskillful operation of the UGFNA for thyroid nodules less than $5 \mathrm{~mm}$ in diameter. ${ }^{13}$ Nodules less than $1 \mathrm{~cm}$ in size should be followed up closely according to the ATA guideline, ${ }^{13,18}$ which is not well accepted in China. Two cases of FN/SFN were proved to be follicular carcinoma (FTC), and one was proved to be follicular tumor of uncertain malignant potential, which indicated the difficulty to cytologically differentiate atypical follicular adenoma from minimally invasive FTC. ${ }^{19}$

Fifteen of 21 cases of AUS/FLUS were proved to be histologically malignant. These results were different from another study in China, as shown in Table 2. The risks of malignancy (ROMs) of AUS/FLUS and suspicious for malignancy were nearly the same, likely due to the different diagnostic criteria for the cytopathologist; when there are less than 30 atypical cells, we make the diagnosis of "AUS/FLUS" instead of "suspicious of malignancy." This is an attempt by the pathologist to suggest a repeat FNA instead of diagnostic surgery for such lesions. In the suspicious of malignancy group, there were three cases of hyalinizing trabecular tumor (HTT). As shown in Fig. 1A and B, there were obvious pseudoinclusions in the cell smear of HTT. Although the nuclear clearing is not conspicuous, the cells were of variable size with nuclear atypia and nuclear grooves, which led to the over-diagnosis of PTC. However, filament-like hyalinizing material was found around the tumor cells, suggesting the possibility of HTT, which is classified as borderline tumor in the new World Health Organization classification of endocrine tumors. ${ }^{20}$

Patients in the "nondiagnostic" group will not undergo immediate operation, and repeat FNA is recommended after 3-month follow-up in our practice. Only six cases were followed by operation,

Table 2. Correlation of FNA cytology with histological diagnosis for the 791 cases

\begin{tabular}{lccccccc}
\hline Bethesda category & Nondiagnostic & Benign & AUS/FLUS & FN/SFN & Suspicious for malignancy & Malignancy & Total \\
\hline Cases for surgery & 6 & 90 & 21 & 3 & 200 & 471 & 791 \\
Malignancy on surgery & 0 & 31 & 15 & 2 & 168 & 69 & 457 \\
ROM (\%) & 0 & 34.4 & 71.4 & 66.7 & 973 & 97.0 & 85.1 \\
ROM from Zhang et al. ${ }^{14}(\%)^{a}$ & 27.9 & 7.9 & 45.5 & 75.5 & 98.5 & 100 & 65.5 \\
ROM from Haugen $^{13}(\%)$ & 20 & 2.5 & 14 & 25 & 70 & Not available \\
\hline
\end{tabular}

FNA, fine-needle aspiration; AUS/FLUS, atypia of undetermined significance or follicular lesion of undetermined significance; FN/SFN, follicular neoplasm or suspicious for a follicular neoplasm.







Fig. 1. (A) Cytology of hyalinizing trabecular tumor. The thin green arrow shows nuclear groove, and the thin blue arrow shows pseudoinclusion. The arrowhead shows filament-like hyalinizing material between the tumor cells. (B) Histology of hyalinizing trabecular tumor. The thin green arrow shows nuclear groove, and the thin blue arrow shows pseudoinclusion. The arrowhead shows hyalinizing material around the cell nests.

and none of them was malignant. The ROM of this category was different from that of another practice in China and Dr. Ali's practice in the United Sates. ${ }^{14,21}$ Routine $B R A F^{\mathrm{V} 600 \mathrm{E}}$ mutation analysis was applied to all FNA samples in Dr. Zhang's practice, which could improve the accuracy rate of FNA. ${ }^{14}$ This is likely one reason that the operation rate was low in our practice for patients in the nondiagnostic category. Another reason could be a significant number of patients went to another hospital instead of our hospital because of bed tension or economic issue. Meanwhile, Dr. Zhang's result suggests that routine $B R A F^{\mathrm{V} 600 \mathrm{E}}$ mutation analysis is necessary to improve the efficiency, sensitivity, and specificity of thyroid FNA cytology.

Above all, thyroid FNA is an important part of preoperative diagnosis, but it is still in an early stage in China compared with histopathology. Ultrasound, radiology, physical presentation, and molecular features of thyroid nodules are critical to achieve an accurate cytologic diagnosis. The last decade has witnessed rapid development of thyroid FNA in China. The next decade will offer exciting opportunities, and international exchange and cooperation are necessary for cytologists to develop thyroid FNA to a higher level.

\section{Conflicts of Interest}

No potential conflict of interest relevant to this article was reported.

\section{REFERENCES}

1. Shu YJ, Kan X. Cytopathology in China. Zhonghua Bing Li Xue Za Zhi 2005; 34: 625-7.

2. Liu DG. Cytology development in recent 10 years and the future outlook in China. J Am Soc Cytopathol 2016; 5: 10-3.

3. Liu DG. Importance of cytopathology. Zhonghua Bing Li Xue Za Zhi 2009; 38: 799-805.

4. Shen O, Liu SF, Dawsey SM, et al. Cytologic screening for esophageal cancer: results from 12,877 subjects from a high-risk population in China. Int J Cancer 1993; 54: 185-8.

5. Shen Q, Qiu S, Zhao H. Report on cytopathological diagnosis for esophageal carcinoma screening. Chin J Pathol 1963; 7: 19-24.

6. Li FP, Shiang EL. Screening for oesophageal cancer in 62000 Chinese. Lancet 1979; 2: 804.

7. Peng XJ, Yan XC. Bone tumor cytodiagnosis with fine-needle aspiration: a preliminary report. Chin Med J (Engl) 1983; 96: 611-8.

8. Zhang Y. Review and prospect of fifty years of Chinese clinical cytology. Chin J Diagn Pathol 1999; 6: 252-5.

9. Fan YB, Wu X, Fu ZM, Wu GP. Amplification of the human telomerase gene in liquid-based preparations is associated with cervical dysplasia and carcinoma. Int J Gynecol Pathol 2010; 29: 157-64.

10. Nikiforov YE, Carty SE, Chiosea SI, et al. Impact of the multi-gene ThyroSeq next-generation sequencing assay on cancer diagnosis in thyroid nodules with atypia of undetermined significance/follicular lesion of undetermined significance cytology. Thyroid 2015; 25: 1217-23.

11. Nikiforov YE, Carty SE, Chiosea SI, et al. Highly accurate diagnosis of cancer in thyroid nodules with follicular neoplasm/suspicious 
for a follicular neoplasm cytology by ThyroSeq v2 next-generation sequencing assay. Cancer 2014; 120: 3627-34.

12. Le Mercier M, D'Haene N, De Nève N, et al. Next-generation sequencing improves the diagnosis of thyroid FNA specimens with indeterminate cytology. Histopathology 2015; 66: 215-24.

13. Haugen BR. 2015 American Thyroid Association management guidelines for adult patients with thyroid nodules and differentiated thyroid cancer: what is new and what has changed? Cancer 2017; 123: 372-81.

14. Zhang YZ, Xu T, Cui D, et al. Value of TIRADS, BSRTC and FNABRAF V600E mutation analysis in differentiating high-risk thyroid nodules. Sci Rep 2015; 5: 16927.

15. Liu Z, Song Y, Han B, Zhang X, Su P, Cui X. Non-invasive follicular thyroid neoplasm with papillary-like nuclear features and the practice in Qilu Hospital of Shandong University, China. J Basic Clin Med 2017; 6: 22-5.

16. Zhu Y, Dai J, Lin X, Wu H, Wang T. Fine needle aspiration of thyroid nodules: experience in a chinese population. J Basic Clin Med 2015;
4: $65-9$.

17. Cibas ES, Ali SZ; NCI Thyroid FNA State of the Science Conference. The Bethesda System For Reporting Thyroid Cytopathology. Am J Clin Pathol 2009; 132: 658-65.

18. Haugen BR, Alexander EK, Bible KC, et al. 2015 American Thyroid Association management guidelines for adult patients with thyroid nodules and differentiated thyroid cancer: the American Thyroid Association Guidelines Task Force on Thyroid Nodules and Differentiated Thyroid Cancer. Thyroid 2016; 26: 1-133.

19. Kini SR. Thyroid cytopathology: an atlas and text. Philadelphia, PA: Lippincott Williams \& Wilkins, 2008.

20. Lloyd R, Osamura R, Kloppel G, Rosai J. WHO classification of tumours: pathology and genetics of tumours of endocrine organs. 4th ed. Lyon: IARC Press, 2017.

21. Ali SZ, Cibas E. The Bethesda System for Reporting Thyroid Cytopathology: definitions, criteria and explanatory notes. New York: Springer, 2010. 